\title{
EpiCare - A home care platform based on mobile cloud computing to assist Epilepsy diagnosis
}

\author{
Daniel Callegari, \\ Endrigo Conte, Tiago Ferreto \\ School of Informatics \\ PUCRS \\ Porto Alegre, Brazil
}

\author{
Dênis Fernandes, \\ Filipe Moraes \\ School of Engineering \\ PUCRS \\ Porto Alegre, Brazil
}

\author{
Fernando Burmeister \\ Dell Computers \\ Porto Alegre, Brazil
}

\author{
Rômulo Severino \\ InsCer - Brain Institute \\ São Lucas Hospital \\ Porto Alegre, Brazil
}

\begin{abstract}
Epilepsy is a neurological disorder that affects a significant percentage of the population. Currently, electroencephalogram exams (EEG) are considered a valuable tool to support epilepsy diagnosis. In order to obtain a more trustworthy diagnosis, it is frequently necessary to submit patients to long monitoring periods. This fact, beyond causing discomfort to the patients and their relatives, implies high costs to the health system. This paper presents EpiCare, a platform to assist epilepsy diagnosis based on mobile cloud computing. The platform allows the continuous monitoring of the patient outside the hospital environment, capturing information of EEG recordings, detecting epileptic events and storing data to be reviewed by a specialist.
\end{abstract}

Keywords - epilepsy, EEG, home care, signal processing, mobile, cloud computing

\section{INTRODUCTION}

Epilepsy is a common neurologic disorder, affecting about $2 \%$ of the population, with more than one million epileptics in Brazil [1]. In the Neurology department of São Lucas Hospital (HSL), the largest amount of patients come from the epilepsy ambulatory, representing 200 to 300 appointments each month. Due to the high prevalence and the physical and social consequences that epilepsy brings to patients, we should look for better ways to treat the disease.

The first step in improving epilepsy treatment is to do a precise diagnosis, since epilepsy is not only one disease with one cause and one treatment, but instead a variety of diseases with different treatments that express themselves as epileptic crises [2]. The evaluation from epileptologist physicians is sometimes the only intervention used to elucidate the case. Moreover, electroencephalogram exams (EEG) help in diagnosing epilepsy but they have some practical limitations [3], especially due to the large amount of data that should be manually analyzed.

At first, due to the paroxystic nature of epilepsy, most part of ambulatory registers - lasting in average 30 minutes - are not long enough to identify the characteristic variations in EEG exams. In order to do longer recordings, patients must go to the hospital and stay as long as necessary to capture those variations [4]. That practice is very expensive due to the low number of rooms in the hospital and the need for a team of professionals such as neurologists, nurses and neurophysiologists with time and experience to manually analyze hours of recordings of many patients. It is also not

Study developed by the Research Group of the PDTI 01/2014, financed by Dell Computers of Brazil Ltd. with resources of Brazilian Law 8.248/91. uncommon to have a patient's relative next to the hospital bed pressing a button to identify and register the approximate timestamp of a clinical epileptic event. The doctors can then focus on the most relevant parts of the whole EEG recording, though at the expense of human intervention.

Portable ambulatory EEG exams already exist, but they also require a neurophysiologist expert to analyze hours of data. Besides, most ambulatory EEG equipment are not capable of sending signals to either hospital or doctors when a crisis is occurring, especially in the cases of pre-SUDEP situations. SUDEP is short for sudden unexpected death in epilepsy, a special condition where the patient eventually dies because of the disease [5].

Due to the increasing number of patients and the costs of transport to and from the hospital as well as the costs of hospitalization, the remote care and monitoring of patients is a long and globally expected trend [3][6].

This paper presents EpiCare, a smartphone- and cloud-based platform for long-term remote epileptic patient monitoring, comprising a pre-SUDEP detection alert and a cloud computing environment for EEG signal analysis and spike detection. Through an automated analysis of epileptic EEG recordings, we can provide doctors a more comprehensive and semi-automated decision platform, while providing the patient a more comfortable monitoring experience at home.

In order to overcome the main limitation of long-term EEG and the large amount of data to analyze (e.g. 48 hours or more per patient), we use specialized algorithms for automatic detection of epileptic spikes. The epileptic spikes play a fundamental role in the diagnosis of epilepsy, providing important information to identify, classify and localize the epileptic focus. In addition, a cloud computing platform is used to provide elastic capacity in terms of processing and storage.

\section{EPICARE OVERVIEW}

The EpiCare platform comprises the following components:

- a patient monitoring kit (electrode cap and EEG hardware);

- a smartphone (including specific application);

- $\quad$ an emergency center capable of receiving notifications and provide immediate care; 


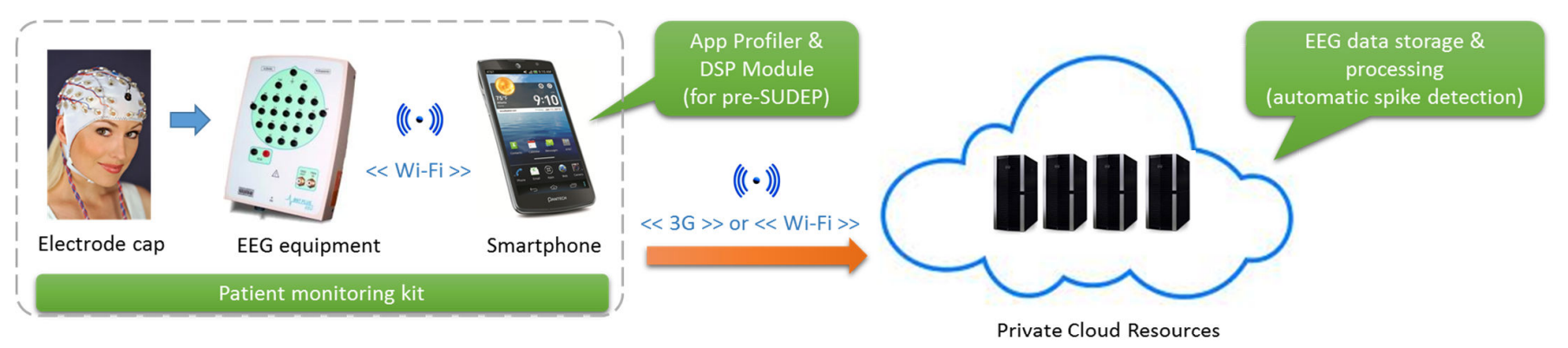

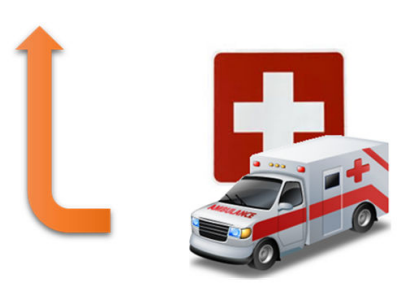

Emergency Center
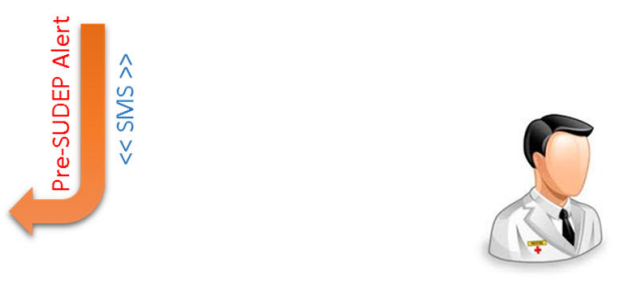

Neurologist

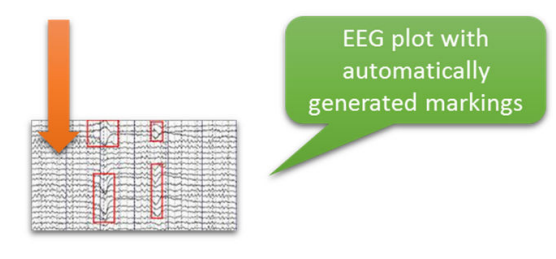

Figure 1 - EpiCare platform

- a cloud computing infrastructure for storage and processing of EEG signals (automated spike detection);

- a medical visualization software that allows doctors to review all identified spikes, so that it is not necessary to manually scan all recorded hours of every patient.

Figure 1 depicts the EpiCare platform. In the top left side, we can see the patient and a home monitoring kit including a smartphone. On the right, we see the cloud infrastructure that stores and analyses EEG data in order to detect spikes. All spikes detected are recorded with their respective timestamps aside the EEG signals; this allows neurologists to focus on relevant parts of the long-term EEG exams.

It is important to note that the system does not tell whether the patient has or hasn't the disease. It works as a spike detection and monitoring tool to assist doctors in their practice. Because there can be false positives during the spike detection phase, the doctors need to agree or discard those automatic markings on the exam. The bottom left of Figure 1 represents a warning that the system raises in the event of a pre-SUDEP situation. The following sections detail the main components (see Figure 2).

\section{A. Monitoring Kit}

The monitoring kit is currently composed of an electrode cap and an EEG rack. The patient wears a cap with embedded sensors disposed in the classical 10-20 system [7]. The cap is wire attached to an EEG rack containing the following components: (a) a $3^{\text {rd }}$ party compatible EEG device and cables; (b) a no-break and power supply; and (c) a Wi-Fi router. This current setup reuses some already available parts provided by the hospital, in order to reduce costs at the present research stage.

The power supply provides energy to the EEG equipment. The EEG device connects to the Wi-Fi router via a standard Ethernet connector and provides filtered live digital data from the sensors, which can be read by the smartphone application.

\section{B. Smartphone application}

The smartphone application is currently executed on an Android based device. The application access the data provided by the monitoring kit through the wireless router.

The application is composed of two pieces of software: (a) a domain specific digital signal processing (DSP) for preSUDEP detection; and (b) a profiling module that monitors the DSP component, the status of the connection to the cloud service, as well as battery, memory and processor usage. This profiling module then decides when it is preferable to perform local or remote processing of the acquired data. This setup provides resilience to the solution.

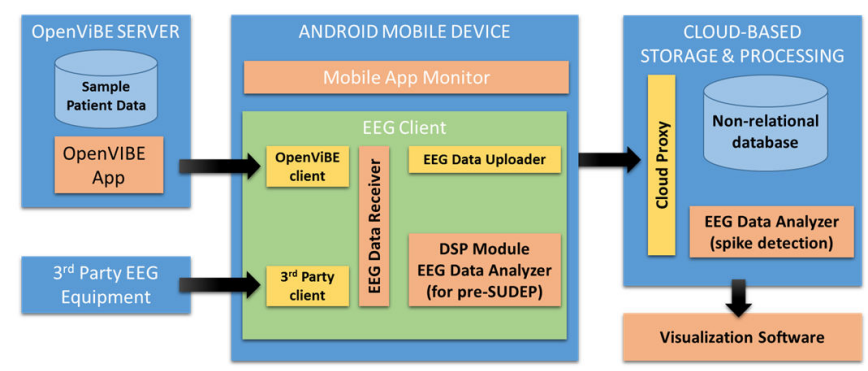

Figure 2 - EpiCare components

The DSP module running on the smartphone looks for brain signals that might lead to a SUDEP event. In such cases, the smartphone sends a message via SMS to a preconfigured telephone number at an Emergency Center, so that help can be provided on time. If connection to the cloud service is not possible, the smartphone stores EEG data until the connection is restored. This application is implemented under the Mobile Cloud Computing (MCC) paradigm. MCC is an emerging computing paradigm that leverages the cloud computing resources to Smart Mobile Devices (SMD) [8]. 
MCC uses cloud storage services for providing online storage, and cloud processing services for augmenting processing capabilities of SMDs. Processing capabilities of SMDs are extended by outsourcing computational intensive components of the mobile applications to cloud datacenters. Although executing heavy tasks on cloud infrastructure could be beneficial, always delegating execution to remote servers might not be advantageous and, in some cases, more resources are spent sending and receiving jobs over the network. For this reason, the mobile device should be intelligent enough to decide whether outsourcing computation is beneficial or not [9]. Thus, our approach includes a software profiler running inside the smartphone in order to manage where the data processing will occur [10].

In order to address this tradeoff, we are improving our model to decide whether some information is better processed localy or else remotely by the cloud. The model comprises a set of variables (such as data size and type) to tune it for each application as well as take into account important network issues, such as latency and cost for both the infrastructure (computational costs) and the final users (monetary costs). By monitoring battery autonomy, application performance, the size and the nature of the data to be processed and considering networking issues, one must be able to make this decision. The first version of the model is already working and laboratory tests are being performed.

\section{Emergency Center}

The emergency center is responsible for dealing with critical situations. It receives notifications from the smartphone, which happens when a pre-SUDEP event is identified. Fast response is mandatory since in this situation the patient can actually die if care is not provided shortly.

\section{Cloud computing infrastructure}

As a model that enables ubiquitous network access to a pool of computing and networking services, Cloud Computing provides computational resources in an on-demand, pay-asyou-go manner through minimal interaction with the service provider [11]. This sort of utility computing infrastructure offers a scalable environment to store and also process longterm EEG signals from many patients simultaneously.

EpiCare stores data on a non-relational database system (i.e. MongoDB), which maximizes throughput, scalability and data model flexibility [12]. Such databases can be scaled to provide high level of parallelization on simple queries supporting incoming data from several patients without losing performance. The platform uses a private cloud, which might be located inside the hospital. This private cloud is implemented using the OpenStack platform [13].

\section{E. Automated spike detection}

Paroxysmal interictal events in EEG are distinctive signatures of epilepsy. These events are epileptiform discharges that occur between seizures and characterize an epileptic symptom. This abnormality consist mainly of abrupt high amplitude peaks (or spikes) and unexpected cerebral rhythm changes in EEG recordings. The traditional definition of spike is based on its amplitude, duration, sharpness, and emergence from the background signal [14]. These epileptic spikes play a fundamental role in the diagnosis of epilepsy, providing important information to identify, classify and localize the epileptic focus.

We investigated the most recent and relevant studies addressing automated spike detection techniques. From 80 selected papers from a literature review, we categorized almost 50 according to five indicators of relevancy (feature extraction method, classification method, dataset, publication year, and accuracy). A comprehensive review of those papers provided us underlying evidences to indicate the wavelet-based methods as the most likely efficient procedures in terms of feature extraction ( $\sim 40 \%$ in our literature review sample) [19].

The stage of spike detection involves defining rules to evaluate spike candidates. Common approaches are based on thresholds and/or learning machines. Often, statistical analysis is applied to determine an adequate threshold. In the same way, artificial neural networks (ANN) are usually employed as general classifiers. Based on different architectures and algorithms, an ANN is able to deal with both raw- and featurebased methods.

The relevancy of feature extraction in automatic detection and classification of epileptic spikes was highlighted by many authors. Song et al. [15] stated that for an effective epilepsy diagnosis model, appropriate methods are required. The authors argue that feature extraction is often applied to complex, high dimensional and multivariate data. Likewise, Tamil et al. [16] remarked that the suitable feature extraction methods are useful to facilitate the representation and interpretation of the data. Therefore, extraction and selection of features play a critical role in detection and classification tasks.

Techniques based on Wavelet Transforms are widely employed in applications where it is necessary to inspect the signals for time and frequency analysis [17]. The main benefit in using wavelets for EEG analysis relies on its multi-resolution ability. This approach provides useful perspectives for EEG signal processing, such as data compression, de-noising and feature extraction [17]. By using wavelets we are able to decompose the EEG signal in sub-bands, capture frequency and time information from low and/or fast transients [15]. Based on the above, we are addressing the spike detection task by using a Wavelet Transform approach, although complementary methods, such as statistical and raw data analysis, could eventually be added to the detection algorithm for performance improvement.

\section{F. Medical visualization software}

Neurologists will have access to our visualization component to retrieve any patient's data from the cloud storage. EEG data processed by our algorithm in the cloud will contain automatically generated markings of detected spikes, so that neurologists can focus on the most important time periods of all recordings, thereby decreasing the time to diagnose the disease. In this review process, physicians can accept or reject each automatic marking, allowing them to base their diagnosis on the whole EEG exam. 


\section{EVALUATION}

The strategy used to evaluate of our prototype was divided in three steps. At first, we are using an emulated environment to identify possible timing constraints and evaluate networking issues. In this step, we use a software called OpenViBE [18] to emulate the patients being monitored. This software enables the loading of real patients' data and the emulation of an EEG monitor used by a patient. The second step will be executed in a controlled environment with real patients. The platform will be tested in the hospital along with the current approach, with the support from the São Lucas hospital and the InsCer Brain Institute.

The performance of our platform will be evaluated, especially in terms of the automatic spike detection; current prototype tests in MATLAB present sensitivity near $90 \%$ by using both raw data and Daubechies' wavelet coefficient as the inputs for a backpropagation multilayer perceptron neural network. The last evaluation step involves using the platform in a patient's home. We intend to evaluate the reduction of costs for both the patient and the hospital, as well as the increase on the number of simultaneous exams that can be performed per month. We will also survey patients and their relatives regarding the convenience of our solution compared to what is currently provided by the hospital. Finally we intend to evaluate the social impact of the solution in real world scenarios in the next years, since some patients come from the Brazilian unified health system (SUS).

\section{FINAL REMARKS AND FUTURE WORK}

Epilepsy disorders are difficult to diagnose and treat. Sometimes long term EEG exams at the hospital are the only resource applied to diagnose the disease. The average local costs for inpatient monitoring is currently USD 1,000.00 a day per patient. Our approach intends to reach more patients, reduce costs, and provide neurologists with a more comprehensive set of data to help in the diagnostics.

At present, some initial results were obtained in both main research areas. In the mobile cloud computing field, the software profiler already works on gathering real time information from a running application on Android. We are now working on the decision system which chooses whether to run a task in the smartphone or in the cloud.

Regarding the remote care of epilepsy patients, we are applying techniques based on wavelet transforms in order to identify spikes in EEG exams. The next steps are to improve and work on the parallelization of the signal processing component running on the cloud infrastructure. This will work by adding or removing processing units according to the number of exams being processed at each time. As another benefit of this research, the spike detection algorithm can also be applied to the exams already being taken at the hospital.

This work presents new findings that build on top of our previous research [10] as an interdisciplinary group of computer scientists, engineers, managers and physicians. Besides the relevant social and economic benefits, the proposed platform brings an excellent opportunity for the development and evaluation of several approaches to signal processing, pattern recognition as well as for mobile systems based on cloud computing concepts.

\section{ACKNOWLEDGMENTS}

We would like to thank our partners at the Brain Institute of the São Lucas Hospital at PUCRS University in Brazil and EMSA Equipamentos Médicos Ltda. for their support on this research.

\section{REFERENCES}

[1] A. Melo; E.M. Yacubian; M.L. Nunes. "Crises epilépticas e epilepsias ao longo da vida: 100 questões práticas”, São Paulo, Brazil, 2006.

[2] C.P. Panayiotopoulos. "A clinical guide to epileptic syndromes and their treatment", Bladon Medical Publishing, 2nd. revised edition, 2010.

[3] J. Askamp, M.J. A. M. van Putten. "Mobile EEG in epilepsy". International Journal of Psycology, vol. 15, 2013.

[4] J.J. Alix; R.H. Kandler; S.R. Mosdekor. "The value of long term EEG monitoring in children: A comparsion of ambulatory EEG and vídeo telemetry". Seizure, April, 23. pp. 1059-1311, 2014.

[5] P. Ryvlin; L. Nashef; T. Tomson. "Prevention of sudden unexpected death in epilepsy: a realistic goal?", Epilepsia, May, 2013.

[6] J. Zhou; R.J. Schalkoff; B.C.Dean; J.J. Halford. "A study of morphologybased wavelet features and multiple-wavelet strategy for EEG signal classification: Results and selected statistical analysis", 35th Annual International Conference of the IEEE, EMBC, 2013.

[7] American Clinical Neurophysiology Society. "Guidelines for standard electrode position nomenclature. American Journal of Electroneurodiagnostic Technology”, 46(3):222-5, Sep., 2006.

[8] M. Shiraz, A. Gani, R. H. Khokhar, and R. Buyya, "A Review on Distributed Application Processing Frameworks in Smart Mobile Devices for Mobile Cloud Computing," IEEE Communications Surveys \& Tutorials, vol. 15, no. 3, pp. 1294-1313, Jan. 2013.

[9] E. Cuervo and A. Balasubramanian, "MAUI: making smartphones last longer with code offload," 8th International Conference on Mobile Systems, Applications, and Services, pp. 49-62, 2010.

[10] D. A. Callegari; L. C. Jersak; and A. C. da Costa, "Technical Trends and Challenges in Mobile Health: A Systematic Review of Recent Available Literature," Proceedings of the 15th International Conference on Enterprise Information Systems, vol.2, pp. 444-454, 2013.

[11] M. Armbrust, I. Stoica, M. Zaharia, A. Fox, R. Griffith, A. D. Joseph, R. Katz, A. Konwinski, G. Lee, D. Patterson, and A. Rabkin, "A view of cloud computing," Communications of the ACM, vol. 53, no. 4, p. 50, Apr., 2010.

[12] S. Tiwari, "Professional NoSQL". John Wiley \& Sons, Inc., p. 384, 2011.

[13] “OpenStack Home Page.” [Online]. Available: www.openstack.org.

[14] M. Latka, Z. Was, A. Kozik, and B. West, "Wavelet analysis of epileptic spikes," Physical Review. E, pp. 1-6, 2003.

[15] Y. Song and J. Zhang, "Automatic recognition of epileptic EEG patterns via Extreme Learning Machine and multiresolution feature extraction". Expert Systems with Applications, vol. 40, no. 14, pp. 5477-5489, Oct., 2013.

[16] E. Tamil, H. Radzi, M. Idris, and A. Tamil, "A Review on Feature Extraction \& Classification Techniques for Biosignal Processing (Part II: Electroencephalography)". 4th Kuala Lumpur International Conference on Biomedical Engineering, vol. 2, pp. 113-116, 2008.

[17] H. Goelz, R.D. Jones, P.J. Bones. "Wavelet analysis of transient biomedical signals and its application to detection of epileptiform activity in the EEG”. Clinical electroencephalography, 31:181-191, Oct., 2000.

[18] Y. Renard, F. Lotte, G. Gibert, M. Congedo, E. Maby, V. Delannoy, O. Bertrand, and A. Lécuyer, "OpenViBE: An Open-Source Software Platform to Design, Test, and Use Brain-Computer Interfaces in Real and Virtual Environments," Presence Teleoperators Virtual Environ., vol. 19, no. 1, pp. 35-53, Feb., 2010.

[19] F. Moraes, D. Callegari. "Automated Detection of Interictal Spikes in EEG: A literature review". PUCRS Tecnical Report TR081. Available: www3.pucrs.br/pucrs/files/uni/poa/facin/pos/relatoriostec/TR081.pdf 